\title{
Creencias y disposiciones de los formadores de profesores de matemáticas en Chile, respecto del enfoque de formación por competencias en el ámbito evaluativo ${ }^{1}$
}

\author{
Alonso Quiroz Meza²
}

RESUMEN

La implementación del Enfoque de Formación por Competencias (EFC) ha implicado no pocos cambios en los programas de formación inicial docente, comenzando por lo curricular y aprestándose a entrar a las aulas con el rediseño de la docencia. En el ámbito de la evaluación, sin embargo, no se observan cambios respecto a las formas tradicionales de evaluar. ¿Qué factores inciden en que los formadores de profesores no estén implementando estrategias de evaluación acordes con el EFC? El presente artículo da cuenta de las creencias y disposiciones de los formadores de profesores de matemáticas, respecto del EFC en el ámbito de la evaluación. Mediante un cuestionario, validado por expertos y prueba piloto, aplicado a 56 formadores de profesores, que realizaban cursos de matemáticas en el primer año de carreras de Pedagogía en Matemáticas a lo largo de todo el país, se pudo constatar que la mayor parte de los encuestados valora positivamente el EFC. Sin embargo, la implementación de dicho enfoque en el ámbito evaluativo es escasa o nula, debido a un desconocimiento de estrategias adecuadas para evaluar competencias matemáticas específicas. Urge, por tanto, generar propuestas adecuadas y viables, de estrategias evaluativas en esta dirección.

I Estudio realizado en el marco de la tesis doctoral "Análisis de las estrategias para evaluar las competencias matemáticas específicas que ponen en juego los formadores de profesores de educación media en Chile. Magíster en Educación Matemáticas. Profesor Titular. E-mail: aquiroz@ucsh.cl

2 Universidad de Sevilla. 


\section{Problema y objetivo del estudio}

En la formación inicial de docentes de matemáticas en Chile, se observa que sus planes de formación describen competencias a desarrollar, no obstante, la formación disciplinar matemática no considera la implementación de estrategias evaluativas que permitan dar cuenta de su desarrollo. De esta forma, se produce un divorcio entre la intención formativa declarada en los perfiles de egreso de la formación inicial docente en matemáticas y la evaluación que efectivamente ponen en juego los formadores de profesores en las asignaturas de matemáticas que realizan en los centros de formación del profesorado de educación media, lo cual afecta a la calidad de la formación inicial docente (Pastor, 2004).

En el ámbito de la Educación Matemática, existe escaso conocimiento y poca valoración de las competencias, así como de su evaluación (Torres, Peralta \& Toscano, 2015). Lo anterior se refleja en los bajos resultados obtenidos por los egresados de carreras de pedagogía en matemáticas en la Prueba Inicia, instrumento diseñado para evaluar el nivel de desempeño de los egresados respecto de los estándares para la formación inicial docente que fueron definidos por el gobierno de Chile el año 2012. Por otro lado, no existen estudios a nivel nacional acerca de la implementación del EFC por parte de los formadores de profesores de matemáticas ni de las estrategias que éstos utilizan para evaluar las competencias matemáticas específicas que eventualmente ponen en juego en las aulas universitarias. Lo anterior implica que cada institución formadora de profesores lo hace a su manera, generando una diversidad de propuestas formativas que afecta negativamente a la calidad de la formación profesional docente.

La investigación tiene por objetivo describir las disposiciones o creencias que tienen los formadores de profesores de matemáticas de educación media en Chile, acerca del enfoque de formación por competencias y las estrategias para evaluar las competencias matemáticas específicas que ponen en juego en asignaturas iniciales de matemáticas. 


\section{Marco referencial}

Los grandes temas que aborda a este estudio y sus contextos son: los formadores de profesores de matemáticas de educación media, el enfoque de formación por competencias, la competencia matemática para la enseñanza, la evaluación para la mejora educativa y la evaluación de competencias matemáticas.

\section{Los formadores de profesores}

Un buen desempeño de un profesor de matemáticas depende de muchos factores entre los que se encuentra el haber tenido una buena formación de parte de sus formadores. En efecto, existe una correlación entre la preparación profesional de los docentes y sus prácticas en clases (Villegas, 1998), por tanto, la insuficiente preparación de los docentes (Vaillant, 2002) hace volver la mirada hacia los formadores, constatando que en América Latina el conocimiento pedagógico de los propios formadores ha sido poco explorado (Messina, 1999). Aún existe la creencia que para ser buen formador basta que sepa muy bien lo que tiene que enseñar, lo más lejos de la realidad actual (Vaillant, 2002). Tomando un esquema realizado en Estados Unidos, Beatrice Ávalos (2004) recoge una serie de desempeños de los formadores de profesores que favorecen buenas prácticas docentes en sus estudiantes:

- Imparten enseñanza efectiva, reflexionan y evalúan.

- Demuestran y estimulan el pensamiento crítico y la resolución de problemas.

- Promueven prácticas que refuerzan la comprensión de la diversidad de los aprendizajes.

- Revisan regularmente los cursos para incorporar materiales recientes, incluyendo tecnología.

- Desafían a los estudiantes a ser reflexivos.

- Usan una variedad de innovaciones en la enseñanza, adecuando los objetivos de aprendizaje con las necesidades de los estudiantes y técnicas de enseñanza apropiadas.

- Aplican conocimiento especializado y procesos de investigación. 
- Demuestran comprensión de la influencia del contexto de la escuela y la cultura sobre el trabajo docente.

- Demuestran conocimiento en relación con temas críticos de la educación

Los formadores de profesores son quienes articulan el currículo con los futuros profesores y en tal sentido deben tener la capacidad de actualizarse permanentemente incorporando reflexivamente las nuevas tendencias de la sociedad y la cultura en el ámbito educativo y dado que la tendencia actual es el desarrollo de competencias, necesitamos investigar cómo hacer que los formadores sean competentes en su rol profesional, y dado que el principal desempeño de sus estudiantes (futuros profesores) es enseñar, el formador tendrá que proveer las oportunidades para que dichos estudiantes logren tener un adecuado desempeño profesional. Enseñar hoy en día se vincula directamente al otorgamiento de oportunidades para desarrollar competencias, no obstante, a la hora de evaluar se produce una esquizofrenia en los formadores, en la que por un lado muestran a sus estudiantes (futuros profesores) extensos y reiterados discursos en que debe favorecerse la evaluación formativa (que es donde se inserta la evaluación por competencias), pero en la práctica favorecen ampliamente la evaluación calificativa (Pastor, 2004).

\section{Enfoque de formación por competencias}

Los desafíos planteados por la UNESCO para la educación del futuro en una sociedad globalizada y del conocimiento, implican una serie de cambios sustantivos en todo el proceso educativo: curriculares, metodológicos, evaluativos, de infraestructura y de gestión administrativa. Se trata de mirar estos aspectos desde una nueva perspectiva, dado que el enfoque tradicional ha mostrado enormes deficiencias en relación con los requerimientos tanto productivos como de inserción social de las personas.

En relación con el diseño curricular, se observa que los modelos analíticos de planeamiento de la enseñanza que se han aplicado hasta la fecha no dan cuenta de una serie de factores que, a partir de los trabajos de Gardner (2000) y Goleman (2006), proporcionan 
evidencias de su importancia a la hora de un buen desempeño laboral. Factores tales como la responsabilidad, la gestión del tiempo, la capacidad de compartir de manera creativa el conocimiento adquirido y el manejo de tecnologías de la información y las comunicaciones, por nombrar sólo algunos, favorecen desempeños exitosos en diversos campos laborales.

En el Espacio Europeo de Educación Superior (EEES) y a propósito de la definición de créditos transferibles, a partir de 1999 se reúnen los ministros de educación de la comunidad europea en Bolonia y posteriormente, el 2000 se incluye la participación cada vez más fuerte y sostenida de las universidades, como agentes válidos para proponer los cambios que se requieren, lo que se expresa en la Declaración de Bolonia (I999). Posteriormente, en la Declaración de Praga (200I) se introducen algunas líneas adicionales, una de las cuales señala: "El aprendizaje a lo largo de la vida como elemento esencial para alcanzar una mayor competitividad europea, para mejorar la cohesión social, la igualdad de oportunidades y la calidad de vida" (p. 9). Este nuevo enfoque denominado "Formación por Competencias" se diferencia del anterior por el tipo de mirada que se da a los procesos, incorporando aspectos de una mirada analítica, a una visión más amplia del aprendizaje, en donde la síntesis, la articulación, lo dinámico y el protagonismo de los sujetos que aprenden, pasa a ser lo relevante.

El concepto de competencia nace de una esfera empresarial, va evolucionando con el tiempo hasta llegar a transformarse en un mega-concepto, expandiéndose a prácticamente todas las esferas del quehacer humano. Etimológicamente, el término competencia proviene de dos palabras del latín: [...cum y petere:-capacidad para concurrir-, coincidir en la dirección, en si significan -poder seguir el paso- por lo que una competencia consistiría en seguir en un área determinada, supone una situación de comparación directa y situada en un momento determinado...] (García \& Tobón, 2008) (p. I8).

Del análisis de las distintas descripciones del concepto de competencia (Segura, 2008) se puede observar que su evolución a lo largo de la historia se ha producido conforme a tres enfoques principales: $U_{n}$ 
primer enfoque orientado a las empresas cuyo centro son las tareas que desarrollar. Un segundo enfoque orientado a la excelencia profesional cuyo centro es el perfil que lograr. Un tercer enfoque orientado a la preparación del ser humano para la vida, cuyo centro es la visión holística y compleja.

La tabla I muestra los diferentes conceptos de competencias y el lugar en donde se encuentran de acuerdo con los criterios señalados.

Tabla I. Características de las competencias según distintos énfasis

\begin{tabular}{|c|c|c|}
\hline CENTRO & ORIENTACIÓN & CONCEPTO \\
\hline Tareas & Empresas & $\begin{array}{l}\text { Conjunto de características inherentes a un } \\
\text { individuo que incluyen habilidades, destrezas } \\
\text { y conocimientos, así como la disposición } \\
\text { a aplicarlas de acuerdo a las demandas del } \\
\text { medio laboral, logrando un desempeño } \\
\text { efectivo para producir una mayor rentabilidad. }\end{array}$ \\
\hline Perfil & $\begin{array}{l}\text { Excelencia } \\
\text { profesional }\end{array}$ & $\begin{array}{l}\text { Características subyacentes en el individuo } \\
\text { que están causalmente relacionadas con un } \\
\text { estándar de efectividad y/o a una performance } \\
\text { superior en un trabajo o situación. }\end{array}$ \\
\hline $\begin{array}{l}\text { Visión } \\
\text { holística } \\
\text { y } \\
\text { compleja }\end{array}$ & $\begin{array}{l}\text { Preparación } \\
\text { para la vida. }\end{array}$ & $\begin{array}{l}\text { Compleja estructura de atributos (conoci- } \\
\text { mientos, actitudes, valores y habilidades) } \\
\text { necesarios para el desempeño de situaciones } \\
\text { específicas con énfasis en el uso o manejo que } \\
\text { el sujeto debe hacer de lo que sabe, no del } \\
\text { conocimiento aislado, en condiciones en que } \\
\text { el desempeño sea relevante". } \\
\text { Constituyen procesos complejos que las } \\
\text { personas ponen en acción-actuación-creación, } \\
\text { para realizar actividades sistémicas y resolver } \\
\text { problemas laborales y de la vida cotidiana, } \\
\text { con el fin de avanzar en la autorrealización } \\
\text { personal, vivir auténticamente la vida y } \\
\text { contribuir al bienestar humano. Integran } \\
\text { el saber hacer (aplicar procedimientos y } \\
\text { estrategias) con el saber conocer (comprender } \\
\text { el contexto) y el saber ser (tener iniciativa } \\
\text { y motivación), con los requerimientos } \\
\text { específicos del contexto en continuo cambio, } \\
\text { con autonomía intelectual, conciencia crítica, } \\
\text { creatividad y espíritu de reto. }\end{array}$ \\
\hline
\end{tabular}

Fuente: Elaboración propia (2015). 


\section{La competencia matemática para la enseñanza}

Uno de los roles esenciales del formador de profesores es otorgar oportunidades para que sus estudiantes aprendan a ser docentes o más específicamente aprendan a enseñar. El futuro profesor necesita saber lo que debe enseñar, pero sobre todo necesita poder enseñarlo (Ávalos, 2004). De acuerdo con Marcelo García (I997), la competencia para enseñar consiste en "la capacidad de actuar en situaciones profesionales, poniendo en juego para ello, conocimientos y habilidades". Así vemos cómo la competencia específica tiene dos componentes: conocimiento y acción práctica, los cuales están dialécticamente relacionados. Dicho de otra forma, la competencia se sitúa a mitad de camino entre el conocimiento y la habilidad, requiriendo saber hacer (habilidad) algo específico (conocimiento) en un contexto dado. Por ejemplo, una persona puede tener habilidad para calcular y no ser competente a la hora de tomar una decisión sobre una inversión por falta de conocimiento de los instrumentos de inversión que existen en el mercado. A la inversa, una persona puede conocer los distintos tipos de instrumentos financieros que existen y no ser competente a la hora de invertir dado que no tiene habilidad para efectuar los cálculos requeridos. Ser competente significa en este contexto conocer los instrumentos de inversión (no todos los instrumentos financieros) y efectuar correctamente los cálculos requeridos (sólo los requeridos para tomar la decisión correcta).

A la luz de lo expuesto, podemos preguntarnos: ¿En qué consiste entonces el enseñar a aprender a enseñar matemáticas? En este punto, los formadores debieran realizar un conjunto de actividades que ofrezcan a sus estudiantes (futuros maestros) oportunidades para aprender a enseñar. Aquí se observan nuevamente las dos componentes de la competencia enseñar (habilidad) matemáticas (conocimiento). En relación con el conocimiento de base para el desarrollo de la habilidad de enseñar, Shulman (2005) distingue los siguientes tipos: El conocimiento del contenido, el conocimiento didáctico general, el conocimiento del currículo, el conocimiento didáctico del contenido, el conocimiento de los alumnos y de sus características, el conocimiento de los contextos educativos y el conocimiento de los aprendizajes esperados. 
Si bien los siete ámbitos de conocimiento profesional docente son esenciales, el conocimiento didáctico del contenido adquiere particular interés ya que identifica los conjuntos de conocimientos distintivos para la enseñanza. En efecto, el conocimiento didáctico del contenido representa la mezcla entre materia y didáctica por la que se llega a una comprensión de cómo determinados temas y problemas se organizan, se representan y se adaptan a los diversos intereses y capacidades de los alumnos y se exponen para su enseñanza. El Conocimiento Didáctico del Contenido es la categoría que, con mayor probabilidad, permite distinguir entre la comprensión del especialista en un área del saber y la comprensión del pedagogo (Shulman, 2005).

En estos estándares se señala qué conocimientos y habilidades deben demostrar los futuros profesores y profesoras en la disciplina respectiva y cómo ésta se enseña, incluyendo el conocimiento del currículo específico, la comprensión sobre cómo aprenden los estudiantes cada disciplina y la capacidad para diseñar, planificar e implementar experiencias de aprendizaje, así como para evaluar y reflexionar acerca de sus logros (p. I2).

En este caso, se observa la directa vinculación de los estándares disciplinarios con las tres primeras categorías del Conocimiento Matemático para la Enseñanza, a saber: Conocimiento común del contenido, Conocimiento especializado del contenido y Conocimiento horizontal del contenido. En el contexto de esta investigación, nos interesa especialmente que los formadores, mediante estrategias de evaluación pertinentes, desarrollen en sus estudiantes (futuros docentes) la capacidad para evaluar y reflexionar sobre los logros de los que serán en el futuro sus estudiantes.

\section{Evaluación para la mejora educativa}

Para Ángelo (1999):

La evaluación es un proceso orientado a comprender y mejorar el aprendizaje de los estudiantes. Ello implica: hacer explícitas y públicas las expectativas educativas; [...] obtener, analizar e interpretar sistemáticamente evidencias que permitan establecer 
la relación entre el desempeño y los estándares y criterios establecidos [...]. La evaluación ha de permitir a los miembros de una comunidad académica, examinar sus propias premisas y crear una cultura dedicada al aseguramiento y la mejora de la educación superior (p. 3-6).

La evaluación considerada como un medio para favorecer el aprendizaje ha sido poco estudiada, sobre todo en lo que se refiere a la retroalimentación que conlleva de manera sustancial (Evans, 2013). En este sentido es importante señalar que todas las fuentes de retroalimentación deben ser consideradas a la hora de evaluar. Funcionalmente, se puede afirmar que el objetivo de la retroalimentación es constituirse en un puente entre el nivel real del aprendiz y la meta de aprendizaje que se ha propuesto el maestro (Ramaprasad, I983; Sadler, I989). De esta forma, se puede señalar que, junto al programa, la evaluación es uno de los elementos del proceso de enseñanza-aprendizaje más importantes debido a la dimensión de retroacción que proporciona al profesor (Marcelo \& Vaillant, 2009).

La educación matemática ha estado interesada en estudiar los fenómenos relativos a la evaluación de los aprendizajes en la disciplina, apuntando a lo que diferencia la evaluación en matemáticas de la evaluación en otras áreas. Así lo demuestran numerosos estudios realizados por destacados investigadores en el área como Kulm, I990; Webb, I992; Lesh \& Lamon, I992; Niss, I993; Romberg, I989, I995; Rico et al., I997; Moya, I995, 200I; García, S., 2003; García, G., 2003; Remesal, 2005 (citados en Hernández \& Romero, 2008).

Webb (I992) ha planteado la situación sobre la base de las siguientes interrogantes: ¿Es necesaria una teoría diferenciada de la evaluación en matemática? ¿Difiere tanto de la evaluación en otras áreas hasta el punto de que tenga sentido una teoría diferenciada de la evaluación en matemática?

En la búsqueda de algunas de las respuestas que están ligadas al quehacer docente, Wilson (1994) plantea que en el campo de la matemática se le da importancia a lo que se evalúa y, por tanto, la evaluación nos da una pista acerca de cuál conocimiento matemático 
resulta ser de importancia para el docente. Smith \& Wood (2000) afirman que la evaluación conduce hacia lo que los estudiantes deben aprender y que eso puede significar la diferencia entre una aproximación superficial o una aproximación profunda al aprendizaje de la matemática. En este sentido, la evaluación puede ser concebida como una práctica de poder (Batalloso, 2000). "Esto es apoyado desde la mirada de Vigotsky, en el sentido de que todos los procesos psicológicos superiores (comunicación, lenguaje, razonamiento) se adquieren primero en un contexto social y luego se internalizan" (Hernández \& Romero, 2008, p. 44). En este sentido podemos afirmar que una de las formas de democratizar el poder de la evaluación es a través del uso de pares, cuestión que ha sido abordada por diversos autores (Siew \& Kian, 2010).

\section{Evaluación de la competencia matemática}

En el caso de las competencias matemáticas específicas, su evaluación será considerando los indicadores para cada una de las tareas propuestas para su desarrollo, recogiendo información sobre las situaciones o contextos, los contenidos matemáticos involucrados, las habilidades puestas en juego, el nivel de complejidad de las tareas y los recursos materiales de que se dispone al efectuar la evaluación.

Las situaciones o contextos definen los ámbitos en los que se sitúan los problemas, es decir, aquella parte del mundo del estudiante (futuro profesor) en el que se localizan las demandas cognitivas hacia él. Se distinguen diversos ámbitos de acuerdo con la cercanía o lejanía del profesor en formación: personal (por ejemplo, resolver un problema de teselación para el embaldosamiento del jardín de su casa), educacional/profesional (por ejemplo, diseñar escalas de notas usando el modelo lineal), público y científico (por ejemplo, calcular el ritmo de crecimiento de una población de bacterias bajo condiciones dadas). Dichos ámbitos constituyen las situaciones de evaluación bajo el enfoque de competencias.

Por otra parte, la organización de los contenidos matemáticos presentes en los currículos de formación inicial docente ha sido hecha considerando sólo las líneas clásicas de desarrollo de la matemática, 
es decir, álgebra, geometría, cálculo, etc. Sin embargo, en la realidad los problemas se presentan de modo tal que los recursos matemáticos empleados no responden a las líneas antes mencionadas.

Este ejemplo muestra que existe otra forma de organizar los contenidos matemáticos para la enseñanza, cual es la fenomenológica. Dos publicaciones muy conocidas dan cuenta de este tipo de organización de los contenidos matemáticos: On the shoulders of giants: New approaches to numeracy (Steen, 1990) y Mathematics: the science of patterns (Devlin, 1994). Desde la mirada fenomenológica, existen grandes ideas matemáticas que sirven para organizar los conocimientos de la disciplina, entre éstas podemos mencionar las ideas de medición, simetría, visualización, algoritmos y clasificación.

Por otra parte, resulta sorprendente constatar que muchas de las habilidades básicas a desarrollar se dan en perspectivas pares complementarias a objeto de proporcionar dos maneras diferentes de enfocar los mismos problemas (Steen, I990): Numérica y visual, Algebraica y geométrica, Formal y experimental, Abstracta y concreta, Analítica y sintética, Algorítmica y existencial, Conceptual y computacional. Es importante que el formador de profesores de matemáticas proporcione las oportunidades para que sus alumnos (futuros profesores) desarrollen estas perspectivas de análisis de contenidos matemáticos.

Es necesario tener en cuenta, además, que los problemas de la evaluación de los conocimientos matemáticos deben ser planteados desde una dimensión epistemológica, puesto que el objeto de la evaluación del aprendizaje es el mismo objeto de conocimiento que la enseñanza pone en acto. Según Ernest (1994), las diferentes escuelas que han caracterizado la naturaleza del conocimiento matemático a lo largo de las diferentes épocas se pueden constituir en dos grandes grupos que están en correspondencia con las concepciones que ellos tienen sobre la Matemática: la prescriptiva o normativa, y la descriptiva o naturalista. Con todo, la tendencia indica que se ha producido un desplazamiento desde la visión de las teorías matemáticas como productos acabados hacia la actividad matemática entendida como una práctica social; así lo entienden Lakatos, I978; 
Wittgenstein, I987, I98I; Ernest, 1989, I99I, I994 (citados por Hernández \& Romero, 2008).

Por último, desde la mirada de los futuros profesores y de sus formadores, la evaluación se complejiza a partir de las concepciones previas que éstos tienen sobre lo que es la matemática, lo cual tiene incidencia en sus percepciones sobre la enseñanza, el aprendizaje y la evaluación de la misma (Berry \& Sahlberg, 1996; Berry \& Nyman, 2002, en Hernández et al., 2008).

\section{Metodología}

Se trata de un estudio extensivo en el que se aplicó un cuestionario validado por juicio de experto y prueba piloto, a una muestra censal de 56 formadores de todo Chile, que estuviesen realizando cursos iniciales de matemáticas en carreras de pedagogía en educación media, en universidades y carreras acreditadas y con ingreso de estudiantes.

El cuestionario se conformó como una escala Likert con puntuaciones de uno a seis, directas e inversas, de acuerdo a una pauta predefinida y por un Diferencial semántico con puntuaciones de uno a seis directas e inversas. Los ítems del cuestionario recabaron información acerca de los siguientes aspectos a indagar:

- Conocimiento, valoración y grado de implementación del enfoque de formación por competencias en general.

- Conocimiento, valoración y grado de implementación de criterios de evaluación de competencias.

- Conocimiento, valoración y grado de implementación de metodologías de evaluación de competencias.

- Conocimiento, valoración y grado de implementación de instrumentos de evaluación de competencias.

La reducción de variables se efectuó mediante un análisis factorial de correspondencias múltiples, logrando una reducción de 36 variables a II factores componentes principales. Se utilizó el software Spss de análisis de datos estadísticos. 


\section{Resultados}

La dimensión I, "Enfoque de formación por competencias y criterios para su evaluación”, de carácter contextual, nos brinda una visión general acerca de cómo los Formadores de profesores de Matemáticas se aproximan al Enfoque de Formación por Competencias y a los Criterios de Evaluación desde esta mirada.

Los resultados del análisis muestran que la inmensa mayoría de los Formadores reconoce las características generales del Enfoque de Formación por Competencias, principalmente su carácter progresivo, movilizador e integrador de saberes para lograr desempeños eficientes frente a la realización de determinadas tareas que implican el logro de determinados objetivos de aprendizaje en diversos ámbitos del quehacer humano. No obstante reconocer las características esenciales del Enfoque de Formación por Competencias, a la hora de su valoración los Formadores se dividen en sus valoraciones en dos grupos que tienden fuertemente al centro, es decir valoraciones insuficientes y valoraciones buenas hacia el Enfoque. Similar cosa ocurre con la implementación de componentes del Enfoque de Formación por Competencias y de sus Criterios: la mitad de los Formadores implementa y la otra mitad no lo hace. Por último, en relación con la valoración de los criterios de Evaluación desde la mirada de las Competencias se observó que existe un alto porcentaje de Formadores que tiene una suficiente o alta valoración por los Criterios de Evaluación desde la mirada de las Competencias.

Por otra parte, si analizamos el comportamiento de la Dimensión considerando algunos componentes de la caracterización de la muestra, los resultados muestran que las Formadoras reconocen de mejor forma las características del Enfoque de Formación por Competencias y tienden hacia una valoración buena o muy buena de dicho enfoque. Además, son más las Formadoras que implementan componentes del Enfoque respecto de los hombres y lo mismo ocurre con la valoración de los Criterios de Evaluación desde las Competencias.

En relación con la región geográfica en donde trabajan los Formadores, respecto de esta dimensión podemos señalar que los 
Formadores de la Zona Norte muestran un mejor reconocimiento de las características del Enfoque de Formación por Competencias, le sigue la Región Metropolitana y por último la Zona Sur del País. Lo mismo ocurre con la valoración del Enfoque y los Criterios de Evaluación asociados. Así mismo se constata que fuera de la Región Metropolitana es donde más se implementa el enfoque y los criterios de evaluación asociados a él. Por último, en la Región Metropolitana es en donde se concentra la mayor parte de los Formadores que tienen una valoración suficiente o alta de los criterios para evaluar desde la mirada de las competencias.

Si consideramos la distribución de la muestra según la antigüedad en la docencia y la formación de postgrado vemos que los formadores que mejor reconocen las características del Enfoque de Formación por Competencias son aquellos que tienen una experiencia laboral de entre cinco y diez años y que tienen alguna formación de post grado en Educación. Por otra parte, los formadores con menos de cinco años de experiencia docente y que no tienen una formación de postgrado en Educación son los que más valoran el Enfoque de Formación por Competencias y sus criterios asociados. La experiencia docente como la formación de postgrado de los formadores no tiene una incidencia significativa en relación con la implementación de componentes del enfoque.

Por último, se constata que los formadores que al caracterizar las competencias ponen énfasis en la integración de saberes son los que en mayor porcentaje reconocen las características del enfoque, valoran los criterios de evaluación desde ésta mirada e implementan componentes del Enfoque de Formación por Competencias. Por otra parte, cabe señalar que quienes menos reconocen las características del Enfoque de Formación por Competencias, son aquellos que tienen una mirada de las competencias muy ligada al desempeño laboral.

La segunda dimensión, denominada "Métodos e instrumentos para evaluar competencias" recoge las creencias y disposiciones de los formadores de profesores de Matemáticas respecto de aspectos más bien prácticos de la evaluación como son el cómo y el con qué evaluar la adquisición de competencias específicas en Matemáticas. Esta dimensión incluye las variables construidas 5, 6 y 7. 
Luego de efectuar el análisis de cada una de las variables de esta dimensión, se pudo observar que sobre el $80 \%$ de la muestra censal valora positivamente los métodos de evaluación asociados al Enfoque de Competencias y sobre el 70\% de los Formadores tiene una buena o muy buena valoración de los instrumentos evaluativos asociados a competencias, sin embargo, se observa que el 57, I4\% de los formadores implementa métodos e instrumentos de evaluación asociados a competencias, porcentaje bajo si se compara con los dos anteriores. Lo anterior significa que existe una visión y disposición positiva hacia el Enfoque de Formación por Competencias de parte de los formadores, sin embargo, al momento de implementar componentes del enfoque en el ámbito evaluativo, se constata que el porcentaje de formadores baja significativamente.

Al pormenorizar el análisis de esta dimensión, vemos que las formadoras, siendo menos en cantidad que los formadores, muestran mejores números en la buena valoración de los Métodos de Evaluación vinculados a las Competencias, en tanto que las formadoras no muestran diferencias significativas con los formadores respecto de las características de los instrumentos de evaluación. Por último, se constata que las formadoras tienen una tendencia hacia una implementación aceptable o buena de las metodologías asociadas a competencias.

Respecto de las características como tipo de jornada académica y denominación del Título Profesional, se constató que los Formadores con Jornada Completa y cuya denominación de Título es "Profesor de Matemáticas”, son los que más positivamente valoran los Métodos de Evaluación asociados a Competencias, así como las características de los instrumentos de evaluación asociados a competencias. Además, estos formadores son los que en mayor número implementan dichos instrumentos.

En relación con la antigüedad en la docencia, los formadores que tienen una antigüedad de diez años o más son los que más implementan instrumentos de evaluación asociados a competencias. Así mismo, los formadores que tienen menos de cinco años o los que tienen entre cinco y diez años muestran un comportamiento 
equilibrado respecto de la implementación de instrumentos. Llama la atención una vez más que los formadores con una antigüedad en la docencia que fluctúa entre los cinco y los diez años son los que muestran una menor valoración positiva hacia las características de los instrumentos de evaluación asociados a competencias.

Cuando se analizan las respuestas en función de la formación de postgrado vemos que hay un equilibrio entre los que implementan instrumentos y los que no lo hacen. Por otro lado, el hecho que la mayoría de los formadores (35 de 56) no tuviese formación de postgrado en Educación no fue motivo para una valoración aceptable o buena de los Métodos de Evaluación asociados a Competencias por parte de éstos.

Por último, se ratifica el hecho de que aquellos formadores que tienen una mirada de las Competencias con énfasis en la integración de saberes son los que más valoran de manera positiva los Métodos de evaluación asociados a competencias, las características de los instrumentos de evaluación asociados a competencias y muestran un alto grado de implementación de los mismos, no obstante existe una alta proporción de formadores que ponen énfasis en la integración de saberes y que no implementan los instrumentos de evaluación asociados a competencias o lo hacen de manera deficiente.

\section{Discusión y conclusiones}

Una competencia corresponde a "un saber actuar complejo que se apoya sobre la movilización y la utilización eficaz de una variedad de recursos". En este sentido, una competencia está bien lejos de un objetivo y no es sinónimo de saber-hacer o de un conocimiento procedimental (Tardif, 20I I).

La mayor parte de los formadores (70\%), prefiere las competencias a los objetivos en el contexto de la educación matemática, cuya caracterización es la de ser profesores de matemáticas varones, de la Región Metropolitana, que no tienen formación especializada en educación, con menos de cinco y más de diez años de experiencia, con jornada completa y que enfatizan la integración de saberes al hablar de competencias. Esto último es significativo ya que los formadores al 
enfatizar la integración de saberes indirectamente están sosteniendo que dicha integración la logran mejor las competencias que los objetivos. Al respecto, Le Boterf (200I) menciona con razón que "es necesario saber hacer reglas de suspensión de la descomposición de recursos” (p. 36).

Le Bortef propone un conjunto de ocho etapas para el desarrollo de un programa de formación por competencias, siendo la primera la determinación de las competencias que componen el programa y la segunda, la determinación del grado de desarrollo esperado por cada una de las competencias al término del programa. Es aquí donde se establecen los criterios para la evaluación de las competencias específicas (Le Bortef, 200I).

Existen indicios claros de implementación de criterios de evaluación de competencias, aun cuando dicha implementación es insuficiente. Los formadores que implementan dichos criterios son mujeres profesoras de matemáticas, de la zona sur, que tienen alguna especialización en educación y que ponen énfasis en la integración de saberes. Lo anterior, a juicio del autor, se debe en gran medida a que los programas de formación les vienen dados desde las jefaturas de carrera o los comités curriculares de las universidades, entonces tratan de implementarlos sin saber a ciencia cierta cuál es su especificidad.

Los problemas que se encuentren y las situaciones inéditas a enfrentar que requieran una nueva configuración de recursos a movilizar, tal vez contribuyan a la evolución constante de las competencias (Le Portier, 20II). Esto es reconocido y valorado por la mayor parte de los encuestados, especialmente por los formadores de la zona norte con alguna especialización en educación, que imparten asignaturas con mezclas temáticas, con entre cinco y diez años de docencia, jornada completa y que valoran fuertemente la evolución del desempeño de los estudiantes independientemente del énfasis que pongan al hablar de competencias.

...De esta forma, se podría afirmar que (las competencias) tienen un carácter integrador porque abarcan conocimientos, procedimientos y actitudes y, además, se construyen con la interrelación de saberes de diferentes ámbitos educativos, donde prima el conjunto integrado de todos ellos más que cada recurso particular (Perales, 2014). 
En términos generales, la inmensa mayoría de los formadores tiene un conocimiento al menos aceptable de la integración de saberes y el desempeño al referirse al tema de las competencias. En particular, la caracterización de los formadores que originan dicho porcentaje es: formadores de género femenino, de la zona norte y que no tienen especialización en educación. Al respecto cabe aclarar que, en este caso, los porcentajes no reflejan el impacto de lo que realmente ocurre, por lo que se consideraron los recuentos en vez de los porcentajes.

Los formadores que imparten asignaturas que mezclan temáticas, con menos de cinco años de antigüedad en la docencia, que son contratados por horas y que manifiestan el énfasis en la integración de saberes, son los que tienen un mayor conocimiento al menos aceptable de la integración de saberes y el desempeño.

La flexibilidad y la adaptabilidad de la competencia justifican plenamente su movilización: todos los recursos disponibles y movilizables no son movilizados en una situación dada, sino solamente aquellos que parecen apropiados en circunstancias precisas. Se trata de una movilización selectiva de recursos (Tadif, 201 I). En este punto, la mayoría de los formadores $(85,71 \%)$ valora la evaluación para movilizar saberes. La caracterización de quienes lo hacen corresponde a mujeres que no tienen especialización en educación, con menos de cinco y más de diez años de experiencia en aula, con a lo menos media jornada y que ponen el énfasis en la integración de saberes. Al autor le queda la duda de si realmente, cuando los formadores están de acuerdo con la evaluación para movilizar saberes, están conscientes que lo clave en dicha movilización es la selección de los recursos a movilizar, de los cuales los saberes son sólo una parte. Esto será indagado en profundidad en la segunda fase de la investigación.

Cabe hacer notar que el análisis precedente está elaborado sobre la base de porcentajes, no obstante, la proporción de formadores que se desempeñan en la Región Metropolitana es muy superior a la de cada una de las zonas norte y sur consideradas por separado. Lo anterior implica que en la mayor parte de las variables los formadores de la Región Metropolitana son los que muestran los mejores índices en términos absolutos. 
Por su parte, la dimensión "métodos e instrumentos para evaluar competencias”, de carácter práctico, describe las creencias y disposiciones de los formadores acerca de cómo evaluar y qué instrumentos les parecen más adecuados para hacerlo.

En términos generales la mayoría de los formadores (78,57\%) es optimista respecto de la factibilidad de aplicar métodos e instrumentos para evaluar competencias, de hecho, poco más de la mitad de los formadores declara implementar métodos e instrumentos de evaluación formativa y la mayor parte de ellos valoran la participación de los estudiantes en la evaluación del desempeño de sus compañeros. Esto último tiene un correlato en la literatura, dado que una de las formas de democratizar el poder de la evaluación es a través del uso de pares, cuestión que ha sido abordada por diversos autores (Siew \& Kian, 2010).

Para Tardif (20II), una competencia no constituye una forma de algoritmo memorizado y practicado repetidamente en vista a asegurar la perennidad y la reproducción, sino un saber actuar muy flexible y adaptable a diversos contextos y problemáticas. Sin embargo, pareciera que los formadores de profesores que trabajan en asignaturas iniciales de matemáticas continúan evaluando mediante pruebas escritas y apuntando a un conocimiento sólo al interior de la disciplina. Por ejemplo, a la hora de valorar los métodos e instrumentos para evaluar la integración de saberes, menos de la mitad de ellos muestra una valoración suficiente o alta, lo cual es corroborado por el hecho de que la inmensa mayoría de los formadores (85\%) aplica escasa o insuficientemente instrumentos para evaluar integración de saberes por parte de sus estudiantes.

Las formadoras son las más optimistas al sostener que es factible la aplicación de instrumentos para evaluar competencias, aplican métodos e instrumentos de evaluación formativa y están de acuerdo con la participación de estudiantes en la evaluación del desempeño de sus compañeros, sin embargo, cabe recordar que éstas son en cantidad la mitad de los hombres. Los formadores con denominación de título "profesor de matemáticas" o "profesor de estado en matemáticas" son los más optimistas en relación con la factibilidad de la aplicación de métodos e instrumentos para evaluar integración de saberes. 
En relación con la zona geográfica en que trabajan los formadores, resulta al menos curioso que las valoraciones se repartan en dichas zonas. Así, si hablamos de la factibilidad de la aplicación de métodos e instrumentos para evaluar integración de saberes debemos irnos a la zona norte, pero si hablamos de la valoración y aplicación de este tipo de métodos e instrumentos, los mayores porcentajes están en la zona sur, y por último si hablamos de la valoración de la participación de estudiantes los mayores porcentajes están en la Región Metropolitana.

Los formadores que tienen alguna especialización en educación implementan métodos e instrumentos de evaluación formativa, tienen una alta valoración de los métodos e instrumentos para evaluar integración de saberes, sin embargo no aplican instrumentos para evaluar dicha integración. En relación a los formadores que no tienen especialización en educación, éstos son optimistas en cuanto a la factibilidad de aplicar métodos e instrumentos para evaluar competencias y valoran la participación de los estudiantes en la evaluación del desempeño de sus compañeros.

En relación con los formadores que tienen más de diez años de experiencia docente, podemos decir que son optimistas en relación con la implementación de métodos e instrumentos de evaluación formativa, y de hecho declaran valorarlos, sin embargo, no aplican ese tipo de instrumentos en las asignaturas que imparten. En cuanto a los formadores con menos de cinco y hasta diez años de antigüedad en la docencia, vemos que también son optimistas respecto de la factibilidad de aplicar métodos e instrumentos para evaluar competencias y valoran la participación de los estudiantes en la evaluación del desempeño de sus compañeros. A este respecto cabe señalar la importancia de la participación de estudiantes en sus procesos de aprendizaje. Calman (1998), citando a Álvarez Méndez (1996), lo expresa de una manera muy clara:

En la medida en que un sujeto aprende, simultáneamente evalúa, discrimina, valora, critica, opina, razona, fundamenta, decide, enjuicia, opta... entre lo que considera que tiene un valor en sí y aquello que carece de él. Esta actitud evaluadora, 
que se aprende, es parte del proceso educativo, que como tal es continuamente formativo (p. 7).

Los formadores que imparten asignaturas que mezclan temáticas son optimistas respecto de la factibilidad de implementar métodos e instrumentos para evaluar competencias y valoran los métodos para evaluar integración de saberes, sin embargo, la aplicación de dichos instrumentos es escasa o nula. Cabe señalar que los formadores que imparten asignaturas de cálculo valoran más la participación de los estudiantes en la evaluación del desempeño de sus compañeros. Esto se condice con lo que plantea la literatura en el sentido que no existe una relación unívoca entre la enseñanza de determinadas áreas o materias y el desarrollo de las competencias (Perales, 20I4). De esta forma se podría afirmar que cada una de las áreas temáticas contribuye a la consecución de diferentes competencias, por lo que podría pensarse que asignaturas que integran temáticas se prestarían de mejor forma para desarrollar competencias.

Por último, debemos constatar que los formadores con jornada completa y cuyo énfasis está en la integración de saberes, son optimistas respecto de la factibilidad de aplicar métodos e instrumentos para evaluar competencias, y valoran la implementación de dichos métodos e instrumentos. Pese a esto la implementación de dichos métodos e instrumentos de evaluación es prácticamente nula. Lo anterior corrobora lo expresado por Solar (2013) en el sentido de que mucho se habla de competencias, pero poco se hace al respecto.

\section{Conclusiones}

Una primera conclusión es que la implementación del enfoque de formación por competencias en Chile se encuentra en su fase inicial. En efecto, tal como lo señala Solar (2010), existen muchos discursos de competencias, pero a la hora de su implementación se observa que es escasa o nula. Lo anterior es corroborado tanto por el estudio cuantitativo que señaló que los formadores en su gran mayoría tienen una mirada intuitiva de las competencias, las valoran, pero sin embargo siguen aplicando estrategias tradicionales de evaluación en las asignaturas iniciales de matemáticas. 
Respecto de la conclusión señalada en el párrafo anterior, los jefes de carrera entrevistados en el estudio de caso señalaron que la implementación de estrategias de evaluación por competencias requiere tener condiciones especiales, como por ejemplo el número de alumnos por asignatura y plataformas informáticas adecuadas para lograr una participación constante de los estudiantes en su proceso formativo. A su vez, es interesante constatar que los grupos focales de estudiantes de pedagogía en matemáticas reconocen la insuficiencia de la evaluación que les es aplicada en la actualidad y sugieren incorporar a la evaluación de asignaturas iniciales de matemáticas, actividades que permitan recoger evidencias de aprendizajes no sólo desde los instrumentos tradicionales de evaluación sino desde las actuaciones correspondientes a su futuro rol de profesores.

Si bien la mayor parte de los formadores sostiene que es razonable incorporar la co-evaluación y la autoevaluación en asignaturas de matemáticas, las aplicaciones de estas prácticas evaluativas están lejos del consenso requerido para una eventual normalización. En efecto el estudio realizado pudo detectar opiniones muy distantes entre los formadores en este respecto, y lo que llama la atención es que los estudiantes de pedagogía, no obstante reconocer que tanto la co-evaluación como la autoevaluación les podría ayudar en términos de obtener buenas calificaciones, manifiestan que no están preparados para ejercer dichas prácticas. La literatura asigna una gran importancia a la autoevaluación y la evaluación de pares como instancias que favorecen los procesos de autorregulación, por tanto, es un desafio para los formadores enseñar a sus estudiantes a evaluar a sus pares y a sí mismos.

\section{Referencias bibliográficas}

Álvarez Méndez, J. M. \& Méndez, J. M. A. (20I I). Evaluar para conocer, examinar para excluir. 4(I). Recuperado el 9 de noviembre de 2016 desde http:// www.fceia.unr.edu.ar/geii/maestria/2013/I0masCelman/\%C3\% 8Ilvarez\%20M\%C3\%A9ndez-Evaluar\%20para\%20conocer\%20 examinar\%20para\%20excluir006.pdf

Angelo, T. A. (1999). Doing assessment as if learning matters most. AAHE Bulletin, 5l(9), 3-6. 
Ávalos, B. (2004). La formación inicial docente en Chile. Documento electrónico recuperado el 10 de marzo de 2017, desde http://www. ub.edu/obipd/PDF\%20docs/Aspectes\%20laborals/Documents/ La\%20Formacion\%20Docente\%20Inicial\%20en\%20Chile.\%20 AVALOS.pdf

Batalloso, J. (2000). ¿Es posible una evaluación democrática?: O sobre la necesidad de evaluar educativamente. En A. Parcerisa (Ed.) Evaluación como ayuda al aprendizaje, (pp.45 54). Barcelona: Graó.

Berry, J. y Nyman, M. (2002). Small-group assessment methods in mathematics. International Journal of Mathematical Education in Science and Tecnbology, 33(5), 64l-649.

Celman, S. (1998). ¿ Es posible mejorar la evaluación y transformarla en herramienta de conocimiento? La evaluación de los aprendizajes en el debate didáctico contemporáneo, 35, 66.

Declaración de Bolonia (Junio de 1999). Declaración conjunta de los ministros europeos de educación. Recuperado el I5 de octubre de 2016 desde http://www.educacion.gob.es/boloniaensecundaria/img/Declaracion_ Bolonia.pdf

Declaración de Praga (Mayo de 200I). Comunicado de la Conferencia de Ministros Europeos responsables de la Educación Superior. Recuperado el 3 de julio de 2015 desde https://www.madrimasd.org/uploads/ acreditacion/doc/rrii_declaracion_praga.pdf

Ernest, P. (Ed.). (1994). Matbematics, education, and pbilosopby: An international perspective London: Falmer Press.

García Fraile, J. \& Tobón, S. (2008). Las competencias en la educación superior: calidad y pensamiento complejo. En García Fraile, J. y Tobón, S.(Coords.) Gestión del currículo por competencias, una aproximación desde el modelo sistémico complejo (pp. 17-39). Lima: A. B. Representaciones Generales.

Gardner, H. (2000). La educación de la mente y el conocimiento de las disciplinas: Lo que todos los estudiantes deberían comprender. Barcelona: Paidós Ibérica.

Goleman, D. (2006). Emotional intelligence. New York: Random House LLC.

Hernández, R. B., \& Romero, A. M. (2008). Una perspectiva crítica de la evaluación en matemática en la Educación Superior. Sapiens: Revista Universitaria de Investigación, 9(l), 35-69.

Le Boterf, G. (200I). Cómpetence et navigation professionnelle. Paris: Éditions d'Organisation.

Marcelo, C. (1997). ¿Quién forma al formador?: un estudio sobre las tareas 
profesionales y necesidades de formación de asesores de Andalucía y Canarias. Revista de educación (313), 249-278.

Marcelo, C. \& Vaillant, D. (2009). Desarrollo profesional docente: Cómo se aprende a enseñar? Madrid: Narcea.

Messina, G. C. (1999). Investigación en o investigación acerca de la formación docente: un estado del arte en los noventa. Revista iberoamericana de Educación (I9), I45-2I0.

Pastor, V. M. (2004). Evaluación, calificación, credencialismo y formación inicial del profesorado: efectos y patologías generadas en la enseñanza universitaria. Revista Interuniversitaria de Formación del Profesorado (5I), $22 \mathrm{I}-232$.

Perales, R. G. (20I4). Diseño y validación de un instrumento de evaluación de la Competencia Matemática: rendimiento matemático de los alumnos más capaces (Tesis Doctoral inédita). Madrid. Universidad Nacional de Educación a Distancia.

Ramaprasad, A. (I983). On the definition of feedback. Bebavioral Science. 28(I), 4-I3.

Sadler, D. R. (1989). Formative assessment and the design of instructional systems. Instructional science, 18(2), 1 19-144.

Segura, R. C. (2008). Mucho que ganar, nada que perder. Competencias: Formación integral de individuos. México D.F: ST Editorial.

Shulman, L. (2005). Conocimiento y Enseñanza: Fundamentos de la nueva reforma. Traducción de Alberto Ide. Revista de currículum y formación del profesorado, 9 (2), I-30.

Siew, L. \& Kian, S. (2010). The effectiveness of peer tutoring in the teaching of mathematics. Vol. 7. Documento recuperado el 5 de agosto de 2015 desde bttp://mjli.uum.edu.my/images/pdf/7mjli/6tbeebbectivenn.pdf

Smith, G. \& Wood, L. (2000). Assessment of learning in university mathematics. International Journal of Mathematical Education in Science and Technology, 3 I, (I) l25 - $\mathbf{l 3 2}$.

Solar, H., Deulofeu, J. \& Azcárate, C. (2010). Competencias de modelización y argumentación en interpretación de gráficas funcionales (Tesis doctoral inédita). Universitat Autònoma de Barcelona. España.

Solar, M. (mayo, 2013). Innovaciones en la formación de profesionales de la educación en Chile. Ponencia presentada en 33rd Annual Seminar of the International Society for Teacher Education. Hong Kong.

Steen, L. A. (Ed.). (1990). On the Shoulders of Giants: New Approaches to Numeracy. Washington DC: National Academies Press. 
Tardif, J. (20I I). Desarrollo de un programa por competencias: De la intención a su implementación. Profesorado:Revista de curriculum y formación del profesorado, I2(3) I - I0.

Torres, A., Peralta, M. \& Toscano, A. (2015). Impacto y productividad de las publicaciones latinoamericanas sobre Matemática Educativa. Biblios: Revista de Bibliotecología y Ciencias de la Información (55), 13-26.

Vaillant, D. (2002). Formación de formadores: Estado de la práctica (No. 658.3124 VI3If). Programa de Promoción de la Reforma Educativa en América Latina. Recuperado el 6 de agosto de 2016 desde http://www.keele. thedialogue.org/PublicationFiles/PREAL\%2025-Spanish.pdf

Villegas, E. (1998). The preparation of teachers in Latin America: Challenges and trends. Human Development Department, World Bank, Latin America and the Caribbean Regional Office. Recuperado el 20 de octubre de 2016 desde http://documents.worldbank.org/curated/ en/I5333I468753024867/pdf/multi-page.pdf

Webb, N. (1992). Assessment of Students Knowledge of Mathematics: Steps Toward a Theory. En: Grouws, D. (Ed.). Handbook of Research on Matbematics Teaching and Learning (pp. 661-683). New York: Macmillan.

Wilson, L. (1994). What gets graded is what gets value. Mathematics Teacher, 87 (6), $4 l$. 